Pacific Journal of Mathematic 


\title{
PARTITION NUMBERS FOR TREES AND ORDERED SETS
}

\author{
ROBERT E. JAMISON-WALDNER
}

In this paper some bounds on the Treberg-type convexity partition numbers of abstract spaces will be presented. The main objective is to show that a conjecture of $J$. Eckhoff relating the Trerberg numbers to the Radon number is valid for a certain class of spaces which include ordered sets, trees, pairwise products of trees and subspaces of these. (Application of the Main Theorem to a certain class of semilattices is given in an appendix.) For ordered sets the results here improve those of $P$. W. Bean and are best possible for general ordered sets.

1. Introduction. To establish terminology, recall that an alignment $[7,8]$ ("algebraic closure system" [2]) on a set $X$ is a family $\mathscr{L}$ of subsets of $X$-to be regarded as "convex" subsets-such that

(A1) $\varnothing, X \in \mathscr{L}$,

(A2) arbitrary intersections of sets in $\mathscr{L}$ are again in $\mathscr{L}$,

(A3) unions of upward directed families of sets in $\mathscr{L}$ are again in $\mathscr{L}$.

The smallest convex set containing a set $S$ is denoted $\mathscr{L}(S)$ and is called the hull of $S$.

A Radon m-partition of $S$ is a partition of $S$ into $m$ subsets

$$
S=A_{1} \cup \cdots \cup A_{m}
$$

such that

$$
\mathscr{L}\left(A_{1}\right) \cap \cdots \cap \mathscr{L}\left(A_{m}\right) \neq \varnothing .
$$

Any point in such an intersection will be called an m-partition point of $S$. It is desirable to allow repeated points in $S$. This can be formalized by letting $S$ be a multiset: each point of $S$ has an integral multiplicity which determines the maximum number of $A_{i}$ 's in which it may be used. (The cardinality $|S|$ of a multiset $S$ is then the sum of the multiplicities of its points.) Sometimes it will be more convenient to think of $S$ as indexed by some set of $|S|$ distinct indices and to associate partitions of the index set with partitions of $S$.

The $m$ th partition number $p_{m}(X)$ of an aligned space $X$ is the smallest integer (if such exists) such that any multiset of cardinality $p_{m}$ of points from $X$ admits a Radon $m$-partition. (The smallest integer $\bar{p}_{m}$ such that any set of $\bar{p}_{m}$ distinct points admits a Radon $m$-partition will be called the restricted mth partition number.) 
Celebrated results of Radon [12] and Tverberg [16] show for the alignment of ordinary convex sets on $\boldsymbol{R}^{d}$ that

(Radon, 1921)

(Tverberg, 1966)
$p_{2}\left(\boldsymbol{R}^{d}\right)=d+2$

$p_{m}\left(\boldsymbol{R}^{d}\right)=(m-1)(d+1)+1$.

In analogy with Tverberg's result and on the basis of numerous abstract examples, Eckhoff [5] suggested that the relation

\section{PARTITION CONJECTURE $\quad p_{m} \leqq(m-1)\left(p_{2}-1\right)+1$}

might be true in any aligned space. Although it is still a long way from establishing this conjecture in general, the main goal of this paper is to show that the partition conjecture holds for a class of spaces including ordered sets and trees. Some weaker bounds on $p_{m}$ will be established in general, and it will be shown that the Partition Conjecture is true when $p_{2}$ equals 2 or 3 .

Following Reay and Sierksma [14], we define the m-core of a multiset $S$ by

$$
\operatorname{core}_{m}(S)=\cap\{\mathscr{L}(S \backslash F):|F| \leqq m\} \text {. }
$$

The $m$ th Helly number $h_{m}(X)$ is the smallest integer (if such exists) such that any multiset $S$ with $|S|>h_{m}(X)$ has nonempty $m$-core. (The restricted $m$ th Helly number $\bar{h}_{m}$ is defined analogously for sets of distinct points.) It follows directly from the definitions that any $m$-partition point of $S$ lies in the $(m-1)$-core of $S$. The converse of this is not true in general, as seen by examining the 2-core of, say, seven points on the unit circle in $\boldsymbol{R}^{2}$. However, as seen in the proof of the Main Theorem, the converse is true for sufficiently large sets of points in trees, and, as shown in the last section, the converse is always true in ordered sets.

The (first) Helly number will be denoted simply by $h(X)$. It is well-known $[11,13]$ that $h(X)$ is the smallest integer $h$ such that:

for any finite family $\mathscr{F}$ of convex sets, if each $h$ or fewer have nonvoid intersection, then all the sets in $\mathscr{F}$ have nonvoid intersection.

The following basic inequalities will be useful in the sequel. They are all immediate consequences of the above definitions and observations:

$$
\begin{aligned}
\bar{p}_{m} \leqq p_{m} ; & \bar{h}_{m} \leqq h_{m} \\
h_{m-1}+1 \leqq p_{m} ; & \bar{h}_{m-1}+1 \leqq \bar{p}_{m} \\
h_{m}=m h_{1} ; \quad & h_{1}=\bar{h}_{1} ; \quad p_{2}=\bar{p}_{2}
\end{aligned}
$$




$$
(m-1) h_{1}+1 \leqq p_{m} .
$$

To obtain the first inequality in (3), one also needs to note that if $S$ is a set with empty 1-core, then the multiset obtained by taking each point of $S$ with multiplicity $m$ has empty $m$-core. Inequality (4) follows than from (2) and (3). The Main Theorem (below) states that (4) is actually an equality for a certain class of spaces. The case $m=2$ of (2) is due to Levi [11].

In Euclidean space with the ordinary alignment, the restricted partition and Helly numbers of all orders agree with their unrestricted analogues. This is due essentially to the continuity of the hull operator and the density of Euclidean space. (Note Lemma 2 in [16].) In discrete situations, the inequalities (1) may be strict for $m>2$. This may happen for trivial reasons: if $X$ is finite and $m>|X|$, then trivially $\bar{p}_{m}=\bar{h}_{m}=|X|+1$ whereas $h_{m}$ and $p_{m}$ always approach $\infty$ as $m \rightarrow \infty$. Sometimes, however, strict inequality occurs in (1) for more interesting reasons: see Theorem 12 in the last section.

2. Examples. Aside from the ordinary convex sets in Euclidean space, there are numerous other examples of alignments of interest. Those of particular pertinence to this paper will be defined and introduced here.

Let $G$ be a graph-for our purpose, a set of nodes with a symmetric, nonreflexive adjacency relation. A set $K$ of the nodes of $G$ will be called path convex provided for each $x$ and $y$ in $K$, all shortest paths in $G$ from $x$ to $y$ lie in $K$. The path convex sets form the path alignment on $G$. A block graph [6] is a graph each of whose blocks (maximal 2-connected subgraphs) is a complete subgraph. A connected block graph will be called a split-tree. Obviously, in any connected graph a path convex set is connected (as a set of nodes). The converse holds precisely for the split-trees. The split-trees are also characterized as those graphs whose path alignment is obtainable by relativization (as described below) of the path alignment on a tree. (These and other results will be presented in a separate study of "tree-like" alignments [9].)

If $(X, \mathscr{L})$ is an aligned space and $Y \subseteq X$, then the restriction of $\mathscr{L}$ to $Y$ given by

$$
\mathscr{L} \mid Y=\{L \cap Y: L \in \mathscr{L}\}
$$

is again an alignment on $Y$ [8]. The process of forming such subspaces $(Y, \mathscr{L} \mid Y)$ is a frequent and natural means of deriving new spaces from old ones. 
If $(X, \mathscr{L})$ and $(Y, \mathscr{C})$ are aligned spaces, the product alignment [8] $\mathscr{L} \times \mathscr{M}$ is defined on the cartesian product $X \times Y$ by

$$
\mathscr{L} \times \mathscr{L}=\{L \times M: L \in \mathscr{L} \text { and } M \in \mathscr{C}\} \text {. }
$$

The determination of various combinatorial invariants of the product from those of the factors is a standing area of interest in axiomatic studies of convexity $[4,13,15]$.

If $X$ is a (partially) ordered set, then a subset $K$ of $X$ is called order convex provided that whenever $x \leqq y \leqq z$ and $x$ and $z$ are in $K$, then $y$ is in $K$. The order alignment on $X$ consists of all order convex subsets of $X$.

3. Some general results. In answer to the question of Eckhoff whether the finiteness of $p_{2}$ implies the finiteness of all the partition numbers, the author presented the proof of the following inequality at the 1976 Oberwolfach Convexity Conference:

$$
p_{m n}=p_{m} p_{n} .
$$

It follows at once from (5) that

$$
p_{m} \leqq\left(p_{2}\right)^{1+\log _{2} m}=p_{2} m^{\log _{2} p_{2}} .
$$

Thus for any aligned space, $p_{m}$ as a function of $m$ grows at most polynomially. Sierksma and Reay [14] recently found another relation on the $p_{m}$ 's which yields a better estimate than (5) in case $m=3$. The following is an improvement of both estimates. (The Sierksma-Reay result is the case $n=2, i=1$.)

THEOREM 1. For any aligned space, the partition numbers satisfy

$$
p_{(m-i) n+\varepsilon} \leqq\left(p_{m}-i\right) p_{n}+\varepsilon
$$

where $0 \leqq i \leqq m$ and $\varepsilon=0$ if $i=0$ and $\varepsilon=1$ if $i \geqq 1$.

Proof. Let $S$ be a multiset with $|S|=\left(p_{m}-i\right) p_{n}+\varepsilon$. Suppose first that $i>0$. Select and fix some point $b$ in $S$. As $b$ may occur with multiplicity 2 or more in $S$, we distinguish one copy of $b$-say, think of it as being colored red. Now divide the remaining points of $S$ into $p_{n}$ submultisets $S(j)$ each with $|S(j)|=p_{m}-i$. Let $\bar{S}(j)$ be $S(j)$ with $i$ copies of red $b$ adjoined. Then $\bar{S}(j)$ admits a Radon $m$ partition with $m$-partition point $t_{j}$. In the partition of $\bar{S}(j)$ there will be at least $m-i$ sets which do not contain any red $b$. List these as

$$
S(j, 1), S(j, 2), \cdots, S(j, m-i) \text {. }
$$

Now the multiset $T$ consisting of all the $t_{j}$ 's admits a Radon $n$-partition 


$$
T=T_{1} \cup \cdots \cup T_{n}
$$

with $n$-partition point $z$. For each $\beta$ from 1 to $n$ and each $\gamma$ from 1 to $m-i$, let

$$
A_{\beta \gamma}=\cup\left\{S(j, \gamma): t j \in T_{\beta}\right\} .
$$

Then for each $\beta$ and $\gamma$, the convex hull of $A_{\beta r}$ contains $T_{\beta}$ and hence $z$. Let $B$ consist of the unused points in $S$. Then $B$ contains red $b$ and hence at least one set in the $m$-partition of $\bar{S}(j)$ for each $j$. Thus $T$ and hence $z$ lies in the convex hull of $B$. The $(m-i) n$ multisets $A_{\beta r}$ together with extra set $B$ form the desired partition of $S$.

If $i=0$, the proof is easier since it is unnecessary to pick a base point $b$ and the sets $A_{\beta \gamma}$ (obtained analogously) form the desired $m n$-partition.

As a illustration, the above theorem leads to the following bounds:

$$
\begin{aligned}
& p_{3} \leqq\left(p_{2}-1\right) p_{2}+1=p_{2}^{2}-p_{2}+1 \\
& p_{4} \leqq p_{2}^{2} \\
& p_{5} \leqq\left(p_{3}-1\right) p_{2}+1 \leqq p_{2}^{3}-p_{2}^{2}+1 \\
& p_{6} \leqq p_{2} p_{3} \leqq p_{2}^{3}-p_{2}^{2}+p_{2} \\
& p_{7} \leqq p_{2} p_{4} \leqq p_{2}^{3}
\end{aligned}
$$

If the alignment in question has a finite Carathéodory number, then it is possible to improve the polynomial bound (6) to a linear one. Recall that the Carathéodory number of an aligned space $(X, \mathscr{L})$ is the smallest positive integer $c$ (if such exists) such that for any subset $S$ of $X$ and any point $p$ in $\mathscr{L}(S)$, there is a subset $F$ of $S$ with $|F| \leqq c$ and $p \in \mathscr{L}(F)$.

THEOREM 2. If an aligned space has finite Carathéodory number $c$ and Helly number $h$, then its partition numbers satisfy

$$
p_{m} \leqq(m-1) c h-c+2 .
$$

Proof. Given a multiset $S$ with the above cardinality, select and fix a base point $b$ in $S$ and let $T=S \backslash b$. Define families of multisets

and

$$
\mathscr{F}=\{T \backslash F:|F| \leqq(m-2) c\}
$$

$$
\mathscr{S}=\{(T \backslash G) \cup\{b\}:|G| \leqq(m-1) c\} .
$$

Any $h$ sets in $\mathscr{S}$ have $b$ in common. If we choose any $h$ sets from $\mathscr{F} \cup \mathscr{S}$, not all in $\mathscr{S}$, their intersection can miss at most 


$$
(h-1)(m-1) c+(m-2) c+1=h c(m-1)-c+1
$$

points of $S$, so that there is still one point left in common. If $\mathscr{C}$ is the family of convex hulls of multisets from $\mathscr{F} \cup \mathscr{S}$, then any $h$ of them have a point in common. Since $h$ is the Helly number, there is a point $z$ common to all the convex sets in $\mathscr{H}$. Clearly $z \in \mathscr{L}(T)$, so there is $A_{1} \subseteq T$ with $\left|A_{1}\right| \leqq c$ and $z \in \mathscr{L}\left(A_{1}\right)$. Now $T \sim A_{1}$ is in $\mathscr{F}$, so there is $A_{2} \subseteq T \backslash A_{1}$ with $\left|A_{2}\right| \leqq c$ and $z \in \mathscr{L}\left(A_{2}\right)$. We may proceed thus until $m-1$ multisets $A_{i}$ of $T$ are so chosen. Then

$$
A_{m}=S \backslash\left(A_{0} \cup \cdots \cup A_{m-1}\right)
$$

is in $\mathscr{S}$ and hence contains $z$ in its convex hull. Thus $A_{1}, \cdots, A_{m}$ is the desired Radon $m$-partition.

The above result is a generalization of the Kay and Womble inequality [10] and was also presented by the author at the 1976 Oberwolfach Conference.

There is one other bound on the partition numbers which is sometimes useful. A subset $I$ of an aligned space $(X, \mathscr{L})$ is independent iff $x \notin \mathscr{L}(I \backslash x)$ for each $x$ in $I$. The rank of $X$ is then defined as the supremum of the cardinalities of independent sets. (Note that for $d \geqq 2$, the rank of $\boldsymbol{R}^{d}$ with the alignment of ordinary convex sets is infinite since the unit sphere is an independent set in this alignment. However, the rank of $\boldsymbol{R}^{d}$ with the alignment of all affine flats is $d+1$.)

The rank is an upper bound for both the Helly number and the Carathéodory number. Indeed, if $\operatorname{core}_{1}(S)=\varnothing$, then $S$ must be independent. Furthermore, if $p \in \mathscr{L}(S)$, then by the finitary property of alignments $[2,8]$, there is a finite subset $F$ of $S$ with $p \in \mathscr{L}(F)$. If we choose this $F$ so as to minimize $|F|$, then it is obvious that $F$ must be independent.

Proposition 3. If $X$ is an aligned space with finite rank $r$, then the partition numbers satisfy

$$
p_{m} \leqq(m-1) r+1 \text {. }
$$

Proof. If $S$ is any finite set and $F$ is a subset of $S$ of minimal cardinal such that $\mathscr{L}(F)=\mathscr{L}(S)$, then evidently $F$ is independent. Hence if $X$ has rank $r$, any finite set $S$ in $X$ contains a subset $F$ with $|F| \leqq r$ and $\mathscr{L}(F)=\mathscr{L}(S)$.

Now let $S$ be given with $|S| \leqq(k-1) r+1$. Choose $F_{1} \subseteq S$ so that $\left|F_{1}\right| \leqq r$ and $\mathscr{L}\left(F_{1}\right)=\mathscr{L}(S)$. Now choose $F_{2} \subseteq S \backslash F_{1}$ so that $\left|F_{2}\right| \leqq r$ and 


$$
\mathscr{L}\left(\boldsymbol{F}_{2}\right)=\mathscr{L}\left(S \backslash \boldsymbol{F}_{1}\right) \subseteq \mathscr{L}\left(\boldsymbol{F}_{1}\right) .
$$

Proceeding in this way, we can peel off $m-1$ sets $F_{i}$. There is at least one point $z$ of $S$ that remains, so that we get

$$
z \in \mathscr{L}\left(F_{m-1}\right) \subseteq \mathscr{L}\left(F_{m-2}\right) \subseteq \cdots \subseteq \mathscr{L}\left(F_{1}\right)
$$

This yields the desired $m$-partition.

Should it happen for a space $X$ that

$$
p_{2}=\operatorname{rank}(X)+1 \text {, }
$$

then the above result yields a trivial verification of the Partition Conjecture in $X$. Of course, (7) is a very restrictive equation, but it does hold for a number of interesting spaces. It certainly holds for the order alignment on a totally ordered set (rank2). It also holds for the alignment of flats on any matroid as well as for certain " $p$-adic convexities" on vector spaces over non-achimedean ordered fields. It will also by shown in Proposition 14 of the last section that (7) holds for the order alignment on the power set of a finite set.

We conclude this section by establishing the Partition Conjecture in case $p_{2}$ is either 2 or 3 .

Proposition 4. If $p_{2}=2$, then $p_{m}=m$ for all $m$.

Proof. One observes that $p_{2}=2$ if and only if every pair (and hence every finite number) of nonvoid convex sets has nonempty intersection.

If the points of an aligned space $X$ are convex, then $p_{2}(X)=3$ is equivalent to $\operatorname{rank}(X)=2$, and Proposition 3 can be applied. If points are not convex, a variant of the argument in Proposition 3 must be applied. It depends on the fact that there is only one type of partition (namely, $3=2+1$ ) and hence the method does not extend to $p_{2}>3$.

Proposition 5. If $p_{2}=3$, then $p_{m} \leqq 2(m-1)+1$ for all $m$.

Proof. Let us say that points $x$ and $y$ dominate a set $S$ provided there are points $d_{x}$ in $\mathscr{L}(x)$ and $d_{y}$ in $\mathscr{L}(y)$ such that

$$
\mathscr{L}\left(x^{\prime}, y^{\prime}\right) \cap \mathscr{L}(s) \neq \varnothing
$$

for all $x^{\prime}$ in $\mathscr{L}\left(d_{x}\right)$ and $y^{\prime}$ in $\mathscr{L}\left(d_{y}\right)$ and $s$ in $S$. 
Claim 1. Given $a \in \mathscr{L}(x), b \in \mathscr{L}(y)$, and $c \in \mathscr{L}(z)$. If $a$ and $b$ dominate $c$, then $x$ and $y$ dominate $z$.

This is obvious since $\mathscr{P}(c) \subseteq \mathscr{L}(\boldsymbol{z})$.

Claim 2. Given three points $x, y$, and $z$, some pair of them dominates the third.

Suppose this fails. Since $x$ and $y$ don't dominate $z$, there are $x_{1}$ in $\mathscr{L}(x)$ and $y_{1}$ in $\mathscr{L}(y)$ with

$$
\mathscr{L}\left(x_{1}, y_{1}\right) \cap \mathscr{L}(z)=\varnothing .
$$

By Claim 1, no two of $x_{1}, y_{1}, z$ dominate the third, so there are $y_{2} \in \mathscr{L}\left(y_{1}\right)$, and $z_{1} \in \mathscr{L}(z)$ with

$$
\mathscr{L}\left(y_{2}, z_{1}\right) \cap \mathscr{L}\left(x_{1}\right)=\varnothing .
$$

Again by Claim $1, x_{1}$ and $z_{1}$ don't dominate $y_{2}$, so there are $x_{2} \in \mathscr{L}\left(x_{1}\right)$ and $z_{2} \in \mathscr{L}\left(z_{1}\right)$ with

$$
\mathscr{L}\left(x_{2}, z_{2}\right) \cap \mathscr{L}\left(y_{2}\right)=\varnothing .
$$

But then $x_{2}, y_{2}, z_{2}$ do not admit a 2-partition since

$$
\begin{aligned}
& \mathscr{L}\left(x_{2}, y_{2}\right) \cap \mathscr{L}\left(z_{2}\right) \subseteq \mathscr{L}\left(x_{1}, y_{1}\right) \cap \mathscr{L}(z)=\varnothing \\
& \mathscr{L}\left(x_{2}, z_{2}\right) \cap \mathscr{L}\left(y_{2}\right)=\varnothing \\
& \mathscr{L}\left(y_{2}, z_{2}\right) \cap \mathscr{L}\left(x_{2}\right) \subseteq \mathscr{L}\left(y_{2}, z_{1}\right) \cap \mathscr{L}\left(x_{1}\right)=\varnothing .
\end{aligned}
$$

This contradicts $p_{2}=3$ and establishes the claim.

Claim 3. Any finite set $S$ with $|S| \geqq 3$ is dominated by two of its points.

When $|S|=3$, this follows from Claim 2. Proceeding by induction, pick any $z$ in $S$ and select $x$ and $y$ in $S \backslash z$ which dominate $S \backslash z$. From the definition of domination select $d_{x}$ and $d_{y}$. By Claim 2, two of $d_{x}, d_{y}$, and $z$ dominate the third.

If $d_{x}$ and $d_{y}$ dominate $z$, then it is easy to see that $d_{x}$ and $d_{y}$ dominate $S$. Whence it follows that $x$ and $y$ dominate $S$.

If $d_{y}$ and $z$ dominate $d_{x}$, pick points $\bar{d}_{y}$ in $\mathscr{L}\left(d_{y}\right) \subseteq \mathscr{L}(y)$ and $d_{z}$ in $\mathscr{L}(z)$ as in the definition of domination. We shall show that $y$ and $z$ dominate $S$. Consider $y^{\prime}$ in $\mathscr{L}\left(\bar{d}_{y}\right)$ and $z^{\prime}$ in $\mathscr{L}\left(d_{z}\right)$ and $s$ in $S$. Note first that

$$
\mathscr{L}\left(y^{\prime}, z^{\prime}\right) \cap \mathscr{L}\left(d_{x}\right) \neq \varnothing,
$$

since $d_{y}$ and $z$ dominate $d_{x}$; let $x^{\prime}$ be any point in the above intersection. Now consider 


$$
D_{s}=\mathscr{L}\left(y^{\prime}, z^{\prime}\right) \cap \mathscr{L}(s) .
$$

If $s=x$, then $x^{\prime} \in D_{s}$. If $s=y$, then $y^{\prime} \in D_{s}$. If $s=z$, then $z^{\prime} \in D_{s}$. If $s$ is not one of $x, y, z$, then $D_{s}$ contains

$$
\mathscr{L}\left(y^{\prime}, x^{\prime}\right) \cap \mathscr{L}(s)
$$

which is nonvoid since $x$ and $y$ dominate $S \backslash z$.

The case that $d_{x}$ and $z$ dominate $d_{y}$ is analogous.

The proposition can now be proved by induction on $m$. Given a multiset $S$ with $|S|=2 m-1$, select by Claim 3 two points $x$ and $y$ of $S$ which dominate it. Then for each $s$ in $S \backslash\{x, y\}$, there is a point $s^{\prime}$ in $\mathscr{L}(x, y) \cap \mathscr{L}(s)$. Let $S^{\prime}$ be the multiset of the $2 m-3$ points so chosen. By induction, these possess an $(m-1)$-partition with partition point $z$. Clearly we can lift this partition to an $(m-1)$-partition of $S \backslash\{x, y\}$. But since

$$
z \in \mathscr{L}\left(S^{\prime}\right) \subseteq \mathscr{L}(x, y),
$$

$\{x, y\}$ may be added to the lifted partition to obtain the desired $m$ partition.

The above argument seems a bit tortuous for such a simple case as $p_{2}=3$. In fact, if $X$ is finite a very short argument is possible. If we wish to partition a set $S$, choose for each $x$ in $S$, some $x^{\prime}$ in $\mathscr{L}(x)$, so that $\mathscr{L}\left(x^{\prime}\right)$ is minimal. The restricted alignment on

$$
S^{\prime}=\left\{x^{\prime}: x \in S\right\}
$$

is easily seen to have rank 2. Proposition 3 can be applied and the desired partition lifted to $S$.

We conclude this section with an example which shows that somewhat complicated alignments can have $p_{2}=3$ and that, in general, a simple reduction to the finite case is not possible.

ExAmple. Let $X=N \times N$, where $N$ is the set of positive integers. Call a subset $K$ of $X$ convex provided

(*) whenever $x \leqq y \leqq z$ and $(x, n)$ and $(z, n)$ are in $K$, then $(y, n+1)$ is also in $K$.

Given three points $\left(x_{i}, n_{i}\right), i=1,2,3$, with $x_{1} \leqq x_{2} \leqq x_{3}$, then clearly $\left(x_{2}, n_{1}+n_{2}+n_{3}+1\right)$ is a 2-partition point, so $p_{2}(X)=3$.

Suppose $S$ is any set of four points of $X$ with distinct first coordinates. We claim that for any finite set $F$ containing $S, p_{2}(F)>3$. 
Indeed, let $(p, m)$ be a point of $F$ with maximal second coordinate. There are then two points of $S$ whose first coordinates lie either both strictly above $p$ or both strictly below $p$. Then $(p, m)$ and these two points do not have a Radon 2-partition in $F$ since any of their 2-partition points has second coordinate $m+1$ or more.

Furthermore, $X$ has infinite rank since $N \times\{1\}$ is an independent set. It is also clear that the lattice of convex sets contains no atoms.

4. The main result. If $p$ is a point in an aligned space $X$, then by a copoint at $p$ we shall mean a maximal convex subset of $X \backslash p$. (The terminology is borrowed from matroid theory; the copoints are just the complete irreducibles in the lattice of convex sets.) We shall make use of some copoint intersection properties as defined below:

CIP $(m, k)$ : for each $p$ in $X$, among any $m$ distinct copoints at $p$, there are $k$ with empty intersection.

MaIN Theorem. Suppose that an aligned space $X$ satisfies CIP $(3,2)$ and has finite Helly number $h$. Then the partition numbers of $X$ satisfy

$$
\begin{array}{ll}
p_{m} \leqq 2 m & \text { if } \quad h=2 \\
p_{m}=(m-1) h+1 & \text { if } \quad h \geqq 3 .
\end{array}
$$

It follows that the Partition Conjecture holds for spaces with CIP $(3,2)$. (This is immediate except in the case that $h=2$ and $p_{2} \leqq 3$. But Propositions 4 and 5 confirm the Partition Conjecture in this case.)

In the proof we shall need the fact that a space with $\operatorname{CIP}(3,2)$ has Carathéodory number $\leqq 2$. This is a special case of a more general result below. Indeed, if we note for each $m \leqq n$ and $k \leqq j$ that $\operatorname{CIP}(m, k)$ trivially implies $\operatorname{CIP}(n, j)$, then the result below implies that a space with CIP $(n+1, j)$, where $n \geqq j$, has Carathéodory number at most $n$.

Proposition 6. An aligned space $(X, \mathscr{L})$ satisfies $\operatorname{CIP}(n+1, n)$ if and only if

(1) the Carathéodory number of $(X, \mathscr{L})$ is at most $n$, and

(2) $(X, \mathscr{L})$ satisfies $\operatorname{CIP}(n+1, n+1)$.

Proof. Suppose CIP $(n+1, n)$ holds; then CIP $(n+1, n+1)$ follows trivially. If $p \in \mathscr{L}(S)$, there is a finite subset $F$ of $S$ with $p \in \mathscr{L}(F)$ since the hull operator is finitary [2, 8]. We may select 
$F$ of smallest possible cardinal. Then for each $x \in F$, the convex set $\mathscr{L}(F / x)$ misses $p$ and hence can be extended to a copoint $C_{x}$ at p. If $|F| \geqq n+1$, then some $n$ of these copoints should have empty intersection. But the intersection of any $n$ copoints $C_{x}$ misses at most $n$ points of $F$ and thus must contain a point of $F$ if $|F| \geqq n+1$. Thus $|F| \geqq n+1$ is untenable, and we must have $|F| \leqq n$ as desired.

Conversely, to see that (1) and (2) imply $\operatorname{CIP}(n+1, n)$, let $n+1$ distinct copoints $C_{0}, C_{1}, \cdots, C_{n}$ at a point $p$ be given. If each $n$ of these have nonempty intersection, pick for each $i=0,1, \cdots, n$, a point $x_{i}$ common to all the $C$ 's except $C_{i}$. As any $n$ of the $x_{i}$ 's lie together in some $C_{j}$, it follows from (1) that $p$ is not in the convex hull of $x_{0}, x_{1}, \cdots, x_{n}$, which can thus be extended to a copoint $D$ at $p$. If $D$ were $C_{i}$ for some $i$, then $x_{i}$ would lie in all $n+1$ of the $C$ 's, contrary to (2). Thus $D, C_{1}, C_{2}, \cdots, C_{n}$ are $n+1$ distinct copoints at $p$. But they have the point $x_{0}$ in common, contradicting (2). This forces us to conclude that some $n$ of the $C_{i}$ 's must have had empty intersection as desired.

Proof of the main theorem. We shall actually prove a stronger result. Namely, if $S$ is a multiset of points in $X$ with $|S| \geqq 2 m$ and $p \in \operatorname{core}_{m-1}(S)$, then $p$ is an $m$-partition point of $S$. The main result follows from this and the observation made in the first section that any multiset of cardinality greater than $(m-1) h$ has nonempty $(m-1)$-core.

It will be convenient here to think of $S$ as being indexed by a set $\Gamma$ of $s=|S|$ distinct indices:

$$
S=\left\{x_{v}: v \in \Gamma\right\} .
$$

Make $\Gamma$ into a graph by defining indices $v$ and $w$ to be adjacent provided $p \notin \mathscr{L}\left(x_{v}, x_{w}\right)$. We shall show that $\Gamma$ contains $m$ mutually disjoint pairs of nonadjacent vertices. These can then be lifted to $S$ to obtain an $m$-partition of $S$ with partition point $p$.

First, let us observe that $\Gamma$ has the following properties:

(1) among any three distinct cliques (maximal complete subgraphs) of $\Gamma$, some two are vertex disjoint, and

(2) any clique of $\Gamma$ contains at most $s-m$ vertices.

Assertion (1) certainly holds if one of the cliques is singleton. (One must be a bit wary of the points $z$ in $S$ for which $p \in \mathscr{L}(z)$.) If a clique $K$ is not singleton, then there is a copoint $C_{K}$ at $p$ in $X$ such that $(*)$

$$
K=\left\{v \in \Gamma: x_{v} \in C_{K}\right\} .
$$

Indeed, since $(X, \mathscr{L})$ has Carathéodory number $\leqq 2, p$ does not belong to the convex set 


$$
\mathscr{L}\left(x_{v}: v \in K\right)
$$

which can thus be extended to a copoint $C_{K}$ at $p$. If $x_{w} \in C_{K}$, then for each $v$ in $K$,

$$
\mathscr{L}\left(x_{w}, x_{v}\right) \subseteq C_{K}
$$

so that $w$ is joined to $v$ in $\Gamma$. Thus by maximality of $K, w$ must lie in $K$. Hence $\left({ }^{*}\right)$ holds, and assertion (1) follows from the $\operatorname{CIP}(3,2)$ in $X$. Assertion (2) follows from the fact that $p \in \operatorname{core}_{m-1}(S)$ and that $X$ has Carathéodory number $\leqq 2$.

We shall now show by induction on $m$, that any graph $\Gamma$ with $s \geqq 2 m$ vertices satisfying (1) and (2) contains $m$ mutually disjoint pairs of nonadjacent vertices. The case $m=1$ is obvious since by (2), $\Gamma$ cannot be complete.

Suppose there are at most two cliques of $\Gamma$ with $s-m$ vertices. Let $A$ and $B$ be distinct cliques for which $|A|+|B|$ is maximal. Choose $a \in A \backslash B$. Since $B$ is a clique and $a \notin B$, there is a $b$ in $B$ that is not adjacent to $a$. Let $\Gamma^{\prime}$ be the graph obtained from $\Gamma$ by deleting $a$ and $b$ (and adjacent edges) from $\Gamma$. Then

$$
\left|\Gamma^{\prime}\right|=s-2 \geqq 2(m-1) .
$$

Since distinct cliques of $\Gamma^{\prime}$ are contained in distinct cliques of $\Gamma$, condition (1) still holds in $\Gamma^{\prime}$. Also since $A$ and $B$ are the only candidates for cliques of size $s-m$ in $\Gamma$, any clique of $\Gamma^{\prime}$ can have at most

$$
s-m-1=(s-2)-(m-1)
$$

vertices, so condition (2) holds in $\Gamma^{\prime}$. Thus induction can be applied. Now suppose there are three or more cliques of size $s-m$ in $\Gamma$. By (1), some two of these, say $A$ and $B$, are disjoint. Thus

$$
s=|\Gamma| \geqq|A|+|B|=2(s-m)=s+(s-2 m) .
$$

Since $s \geqq 2 m$, it follows that $s=2 m$ and that $A$ and $B$ partition the vertices of $\Gamma$. If $C$ is a third clique of $s-m=m$ vertices, then since $C$ neither contains nor is contained in $A$ or $B$, there is some $a$ in $A \cap C$ and some $b$ in $B \backslash C$. Note that $a$ and $b$ cannot be joined by an edge, since there would then be three distinct cliques meeting at $a$, contrary to condition (1).

Suppose $D$ is yet another clique of $s-m=m$ vertices. If $D$ and $C$ meet in some vertex $v$, then $v$ lies in either $A$ or $B$ and hence in three cliques, contradicting (1). Thus $D$ and $C$ are disjoint. Whence from cardinality considerations, $D$ is the complement of $C$. It follows that there can be no fifth clique of size $s-m$.

Now if $D$ exists, it contains $b$ (as it was chosen from the complement of $C$ ). Thus by removing $a$ and $b$, we reduce by 1 the size 
of $A, B, C$, and $D$ (if it exists), so that induction can be applied as before.

In order to apply the Main Theorem to the actual evaluation of partition numbers, it is necessary to be able to compute the Helly number. A subset $S$ of an aligned space is said to be free if it is both convex and independent (that is, all subsets of $S$ are convex). A clique is a maximal free set. (Notice that in a connected graph with the path alignment, the cliques in this sense coincide with the cliques of the graph.) Evidently, every clique has empty 1-core, so that the clique number-the supremum of the cardinalities of all cliques-is a lower bound on the Helly number. Under conditions described below, the two are equal. As cliques are often easy to recognize, this provides a method of evaluating the Helly number.

A point $p$ of a convex set $K$ is an extremepoint of $K$ provided $K \backslash p$ is convex. An aligned space $(X, \mathscr{L})$ is extremally detachable (ED) if for any finite set $F$ in $X$, the polytope $\mathscr{L}(F)$ is the convex hull of its extremepoints (which necessarily lie in $F$ ) [7].

THeOREM 7. If $X$ is a finite ED space, then the Helly number of $X$ equals the clique number of $X$.

This is an immediate consequence of the following more general result.

THeOREM 8. Suppose $X$ is a finite ED space and $S$ is a subset of $X$ with core $_{m}(S)=\varnothing$ for some $m$. Then there is a convex subset $S^{\prime}$ of $\mathscr{L}(S)$ with $\left|S^{\prime}\right|=|S|$ and $\operatorname{core}_{m}\left(S^{\prime}\right)=\varnothing$.

Proof. For any $M \subseteq X$ and any $p \in \mathscr{L}(M)$, define

$$
E_{m}(p, M)=\{A \leqq M:|A| \leqq m \text { and } p \notin \mathscr{L}(M \backslash A)\} .
$$

Then $p \in \operatorname{core}_{m}(M)$ iff $E_{m}(p, M)=\varnothing$. Pick $p$ in $\mathscr{L}(S) \backslash S$ so that the family $E_{m}(p, S)$ is minimal with respect to inclusion (minimal cardinality will do this). Let $A \in E_{m}(p, S)$. Since by ED, $p$ is in the convex hull of the extremepoints of $\mathscr{L}(S)$, there must be at least one extremepoint $z$ of $\mathscr{L}(S)$ in $A$. Since any extremepoint of $\mathscr{L}(S)$ must lie in $S, z \in S$.

Let $T=(S \backslash z) \cup p$. Since $\mathscr{L}(S) \mid z$ is convex, it follows that $\mathscr{L}(T) \subseteq \mathscr{L}(S) \backslash z$ so $|\mathscr{L}(T)|<|\mathscr{L}(S)|$.

Now consider $q \in \mathscr{L}(T)$. If $A \in E_{m}(q, S)$, let $A^{\prime}=(A / z) \cup p$. Then $\left|A^{\prime}\right|=|A| \leqq m$. Also $T \backslash A^{\prime}=S \backslash A$, so 


$$
q \notin \mathscr{L}(S \backslash A)=\mathscr{L}\left(T \backslash A^{\prime}\right)
$$

whence $A^{\prime} \in E_{m}(q, T)$. Thus $q \notin \operatorname{core}_{m}(T)$.

Now if $A \notin E_{m}(q, S)$, since $E_{m}(q, S)$ is not a proper subfamily of $E_{m}(p, S)$, there is some set $B$ in $E_{m}(q, S)$ that is not in $E_{m}(p, S)$. Let $B^{\prime}=B \backslash z$. Then $B^{\prime} \leqq T$ and $\left|B^{\prime}\right| \leqq|B| \leqq m$. Note that $p$ is the only point of $T \backslash B^{\prime}$ that is not in $S \backslash B$. But since $B \notin E_{m}(p, S)$, we have $p \in \mathscr{L}(S \backslash B)$. Thus $\mathscr{L}\left(T \backslash B^{\prime}\right) \subseteq \mathscr{L}(S \backslash B)$. Since $q$ is not in $\mathscr{L}(S \backslash B)$, it cannot be in $\mathscr{L}\left(T \backslash B^{\prime}\right)$ either, so $B^{\prime} \in E_{m}(q, T)$ and $q \notin \operatorname{core}_{m}(T)$.

It follows that core $_{m}(T)=\varnothing$. Since $X$ is finite, this process can be carried on until we reach a set $S^{\prime} \subseteq \mathscr{L}(S)$ with $\left|S^{\prime}\right|=|S|$ where $\mathscr{L}\left(S^{\prime}\right) \backslash S^{\prime}$ is empty - that is, $S^{\prime}$ is convex.

As an illustration we give an alternate approach to Doignon's determination [3] of the Helly number of the lattice points in $\boldsymbol{R}^{d}$.

THEOREM [Doignon]. Let $X$ consist of all points in $\boldsymbol{R}^{d}$ with integer coefficients. With the restriction of the ordinary alignment, the Helly number of $X$ is $2^{d}$.

Proof. Since it is easily seen that any subspace of an ED space satisfies ED and since the convex hull in $X$ of any finite set is finite, it suffices by Theorem 7 to show that the clique number of $X$ is $2^{d}$. We apply Valette's argument [3]. If $K$ contains $2^{d}+1$ points, two of them, say $p$ and $q$, are coordinatewise congruent modulo 2 . Thus $r=(p+q) / 2$ is again in $X$. If $r \notin K$, then $K$ is not convex. If $r \in K$, then $K$ is not independent. Hence $K$ is not free and the clique number is at most $2^{d}$.

As the $2^{d}$ vertices of any fundamental cube in $X$ do form a clique, the proof is complete.

5. Trees and split-trees. If $G$ is a split-tree (connected block graph), then it is easily seen that the path alignment on $G$ is ED. Hence by Theorem 7, the Helly number of the path alignment equals the maximum number of points in a clique of $G$. For any node $p$ of $G$, the copoints at $p$ are just the components of $G \backslash p$. Hence the path alignment on $G$ satisfies $\operatorname{CIP}(2,2)$ and thus $\operatorname{CIP}(3,2)$. Therefore the Main Theorem may be applied.

It is a simple matter to see that the bounds in the Main Theorem are achieved among split-trees. For $h=2$, consider $2 m$ nodes all joined to a central node $p$. No proper subset of these extreme nodes admits a Radon $m$-partition. For $h \geqq 3$, take a complete graph on 
$h$ nodes and adjoin at each of its points a path of length $\geqq m$. Choosing $m-1$ points from each of these paths yields a set of $(m-1) h$ points with empty $(m-1)$-core and hence with no $m$-partition points. As these examples involve distinct points, no better bound for $\bar{p}_{m}$ is possible for general split-trees than the bound obtained from the Main Theorem.

To apply the main result to products requires the following observation.

Proposition 9. If $(X, \mathscr{L})$ satisfies $\operatorname{CIP}(m, k)$ and $(Y, \mathscr{M})$ satisfies $\operatorname{CIP}(n, j)$, then the product $(X \times Y, \mathscr{L} \times \mathscr{C l})$ satisfies

$$
\mathrm{CIP}(m+n-1, \max (k, j)) \text {. }
$$

Proof. Any copoint at $(p, q)$ in $X \times Y$ is either of the form $C_{p} \times Y$, where $C_{p}$ is a copoint at $p$ in $X$, or the form $X \times C_{q}$ where $C_{q}$ is a copoint at $q$ in $Y$.

Thus the product of two split-trees satisfies $\operatorname{CIP}(3,2)$, so the Partition Conjecture is true for such spaces. It is also interesting to note that the main result also applies to any subspace of a product of two split-trees, as follows from the next observation.

Proposition 10. If $(X, \mathscr{L})$ has $\operatorname{CIP}(m, k)$ and $Y \subseteq X$, then $(Y, \mathscr{L} \mid Y)$ also has $\operatorname{CIP}(m, k)$.

Proof. Let $C$ be a copoint in $Y$ at a point $p$ of $Y$. Because $C$ is relatively convex, $C=\mathscr{L}(C) \cap Y$, so $p \notin \mathscr{L}(C)$. Hence there is a copoint $C^{\prime}$ in $X$ at $p$ containing $\mathscr{L}(C)$. Since $C^{\prime} \cap Y$ is relatively convex and contains $C$, it follows by maximality of $C$ that $C=C^{\prime} \cap Y$. Thus $m$ distinct copoints in $Y$ at $p$ can be embedded in $m$ necessarily distinct copoints in $X$ at $p$. As some $k$ of the larger copoints have void intersection in $X$, so will the $k$ copoints in $Y$ which they contain.

Of particular interest is the case of the product of two lines: $\boldsymbol{R}^{2}$ with the (Eckhoff) product alignment. The general bounds of Thompson and Hare [15] for the partition numbers of a product in terms of the factors yield estimates for $p_{m}(\boldsymbol{R} \times \boldsymbol{R})$ that an quadratic in $m$. Since the Helly number of the product alignment on $\boldsymbol{R} \times \boldsymbol{R}$ is 2, it follows from the Main Theorem that

$$
p_{m}(\boldsymbol{R} \times \boldsymbol{R}) \leqq 2 m .
$$

That this bound is attained can be seen as follows. 
Let $\pi(i)$ be any permutation of $1, \cdots, 2 m-1$ such that $\pi(m) \neq m$. Let

$$
S=\{(i, \pi(i)): i=1, \cdots, 2 m-1\} .
$$

Suppose $(p, q)$ is an $m$-partition point of $S$. Then $(p, q)$ is in core con-1 $_{m}(S)$. If $p<m$, then the set $H=\{(x, y): x \geqq m\}$ is product convex, contains all but $m-1$ points of $S$, but misses $(p, q)$, contrary to $(p, q) \in$ core $_{m-1}(S)$. Thus $p \geqq m$. By similar arguments, one is led to $p=$ $m=q$. Since $(m, m) \notin S$, each set of the associated $m$-partition of $S$ must contain at least 2 points, for a total of $2 m>|S|$ points. Hence $S$ has no Radon $m$-partition.

Since the points in the above example are distinct, it follows that

$$
\bar{p}_{m}(\boldsymbol{R} \times \boldsymbol{R})=2 m .
$$

Regrettably the Main Theorem does not apply, for $n>2$, to $n$-fold products of lines (or trees), since in general these satisfy CIP $(n+1,2)$ but no stronger CIP. Although nothing better than the Thompson and Hare bounds are known for the higher order partition numbers of large products of general split-trees, it is at least possible to extend Eckhoff's determination [4] of the Radon number $p_{2}$ of a product of lines to a product of trees. For notational convenience, we shall denote the middle binomial coefficient coefficient by mbc $(r)$.

THEOREM 11. For $n \geqq 2$, let $X$ be a product of split-trees $T_{1}, \cdots, T_{n}$ where the Helly number of each $T_{i}$ is at most 3 . If $X$ is endowed with the product of the path alignments, then

$$
p_{2}(X) \leqq \min \{r: \operatorname{mbc}(r)>2 n\} .
$$

It is a bit suprising that the estimate is the same for products of trees as for products of lines. After all, in trees there is much more freedom and usually more than just two "ends". Basically, the idea is to replace the notion of the "ends" of a line by the notion of a hemispace-a convex set whose complement is convex. The following separation axiom will be used in the proof:

$S_{4}$ : if $A$ and $B$ are disjoint convex sets, then there is a hemispace $H$ which contains $A$ but is disjoint from $B$.

This is easily established for the path alignment on split-trees [9]. The feature of hemispaces that is critical for the proof of Theorem 11 is contained in the following lemma.

Lemma. If $T$ is a split-tree with Helly number at most 3, then 
any two hemispaces in $T$ are either comparable, disjoint, or cover $T$.

Proof. Suppose $A$ and $B$ are hemispaces for which the lemma fails. Then, denoting by $A^{\prime}$ and $B^{\prime}$ the convex complements of $A$ and $B$, we see that each of the four intersections

$$
A \cap B, \quad A \cap B^{\prime}, \quad A^{\prime} \cap B, \quad A^{\prime} \cap B^{\prime}
$$

is nonempty. Thus each of the four unions

$$
A \cup B, \quad A \cup B^{\prime}, \quad A^{\prime} \cup B, \quad A^{\prime} \cup B^{\prime}
$$

is a union of two intersected connected sets and hence is connected and thus convex. The intersection of any three of these unions is nonvoid since it contains the intersection of the $A$-term appearing twice with the $B$-term appearing twice. For example,

$$
A^{\prime} \cap B \leqq(A \cup B) \cap\left(A^{\prime} \cup B\right) \cap\left(A^{\prime} \cup B^{\prime}\right) .
$$

Since $h(T) \leqq 3$, the intersection of all four unions should be nonvoid. But direct computation shows that it is empty. This contradiction establishes the lemma.

Proof of Theorem 11. Let $S$ be a multisubset of $X$ with $|S|=r$ where $\operatorname{mbc}(r)>2 n$. Let $S$ be indexed by a set $\Gamma$ of $r$ distinct indices. Let us say that a subset $\Delta$ of indices is killed in coordinate $i$ provided

$$
\mathscr{L}_{i}\left(\pi_{i}\left(s_{\delta}: \delta \in \Delta\right)\right) \cap \mathscr{L}_{i}\left(\pi_{i}\left(s_{\delta}: \delta \notin \Delta\right)\right)=\varnothing .
$$

(Here $\mathscr{L}_{i}$ is the path alignment on $T_{i}$ and $\pi_{i}$ is the $i$ th coordinate projection.) It follows from $S_{4}$ that if $\Delta$ is killed in coordinate $i$, then there is a hemispace $H$ in $T_{i}$ such that

$$
\Delta=\left\{\delta \in \Gamma: \pi_{i}\left(s_{\delta}\right) \in H\right\} .
$$

Our goal is to show that at most two sets of [r/2] indices can be killed in each coordinate. Let us suppose this is not the case for the $i$ th coordinate, and let us introduce the following weight function on subsets $P$ of $T_{i}$ :

$$
w(P)=\left|\left\{\gamma \in \Gamma: \pi_{i}\left(s_{\gamma}\right) \in P\right\}\right| .
$$

If three distinct sets of $[r / 2]$ indices are killed in $T_{i}$, then by $\left(^{*}\right)$ there are three distinct hemispaces $A, B, C$ in $T_{i}$ of weight [r/2] which kill these sets of indices. If, say, $A$ and $B$ were comparable, since they have the same weight, it would follow from $\left(^{*}\right)$ that they kill the same set of indices. But we are assuming this is not the case. Hence $A, B, C$ are pairwise incomparable. 
By the lemma, either $A \cup B=X$ or $A \cap B=\varnothing$. Suppose $A \cup B=X$. If $A \cap C$ were empty, we would then get $C \leqq B$, which is not the case. Thus by the lemma, $A \cup C=X$ and likewise $B \cup$ $C=X$. Therefore if some pair of $A, B, C$ cover $X$, then any pair does. Dually, if some pair of $A, B, C$ are disjoint, then they are mutually disjoint.

But if $A, B, C$ are mutually disjoint, then

$$
r=w\left(T_{i}\right) \geqq w(A)+w(B)+w(C)=3[r / 2]>r,
$$

a contradiction. (For the last inequality, we need $r>3$ which follows from $n \geqq 2$.)

If $A, B, C$ pairwise cover $T_{i}$, note that

$$
r=w\left(T_{i}\right) \leqq w(A)+w(B)=2[r / 2] .
$$

Thus $r$ must be even. Consequently the complements $A^{\prime}, B^{\prime}, C^{\prime}$ all have weight $r / 2$ and are pairwise disjoint. Thus we can derive a contradiction as above.

It follows that if $\operatorname{mbc}(r)>2 n$, then some set of [r/2] indices is not killed in any coordinate and hence, by the definition of the product alignment induces a Radon 2-partition of $S$.

Eckhoff's construction in [4] shows that the above bound is best possible for lines with sufficiently many points and hence for trees of large diameter. For trees of small diameter, improvements are possible.

The behavior of the higher order partition numbers is more complicated and remains to be explored for $n \geqq 3$. The same is true for products of trees whose Helly numbers are more than 3. (One sees easily that the conclusions of both the lemma and Theorem 11 fail in this case.)

6. Ordered sets. If $X$ is a (partially) ordered set, we define

$$
\downarrow p=\{x \in X: x \leqq p\}
$$

for each $p$ in $X$. If $S$ is a multisubset of $X$, then $|S \cap \downarrow p|$ will denote the sum of the multiplicities of the points of $S$ that lie below $p$. Analogous definitions apply to $\uparrow p$.

Note that a point $p$ in $X$ is an extremepoint of $X$ w.r.t. the order alignment iff $p$ is either maximal in $X$ or minimal in $X$. Obviously, at any extremepoint there is only one copoint-namely, its complement. If $p$ is not extreme, then there are two copoints at $p$, namely 
Thus at each point in an ordered set there are at most two copoints, so the order alignment satisfies $\operatorname{CIP}(3,2)$ for trivial reasons. Hence the Main Theorem applies and we get

$$
p_{m}(X)=(m-1) h(X)+1 \text {. }
$$

(Note that if $h(X)=2$, then $X$ must be a chain.) This is an improvement of the result of Bean [1] and in light of (4) is best possible.

When the Helly number is even, say $h=2 n,(8)$ also gives the best (general) bound for the restricted partition numbers. Indeed, let $X$ be a disjoint union of $n$ infinite chains with points on different chains noncomparable. Picking $2(m-1)$ points from each chain yields a set $(m-1) h$ distinct points which admit no Radon $m$-partition.

When the Helly number is odd, it turns out (Theorem 12 below) that a much better bound than (8) holds for $\bar{p}_{m}$. In fact the worst possible behavior in this case is illustrated by adding 2 distinct minimal points to the bottom of the first chain in the preceding example. The resulting ordered set has Helly number $h=2 n+1$. By choosing $m-2$ points from the first chain plus the two new minima plus $m-1$ points from each of the remaining chains, one can obtain a set of $(h-1)(m-1)+1$ points which admits no Radon $m$-partition.

THEOREM 12. Let $X$ be an ordered set with odd Helly number h. Then

$$
\bar{p}_{m}(X) \leqq(m-1)(h-1)+2 .
$$

The proof will use a simple proposition which, in conjunction with (3) of the Introduction, provides a direct proof of (8) without use of the Main Theorem.

Proposition 13. Let $p$ be a point in an ordered space $X$, and let $S$ be a multisubset of $X$. Then the following are equivalent:

(1) $p \in \operatorname{core}_{m-1}(S)$,

(2) $|\downarrow p \cap S| \geqq m$ and $|\uparrow p \cap S| \geqq m$,

(3) $p$ is an m-partition point of $S$.

Proof. (1) $\rightarrow$ (2) If $\downarrow p$ contained $m-1$ or fewer points of $S$, then by definition of the core, $p$ should be in the convex hull of $S \backslash \downarrow p$. But $S \backslash \downarrow p$ its contained in $X \backslash \downarrow p$ which is order convex and misses $p$. Thus $\downarrow p$ must contain at least $m$ points of $S$; likewise for $\uparrow p$.

$(2) \rightarrow(3)$ Suppose $p$ itself occurs with multiplicity $k \geqq 0$ in $S$. 
Then $\downarrow p$ contains at least $m-k$ points of $S$ other than $p$; likewise for $\uparrow p$. Pairing these together in any way yields $m-k$ pairs each having $p$ in its order convex hull. Adjoining $\{p\}$ taken $k$ times yields the desired Radom $m$-partition.

$(3) \rightarrow(1)$ As observed in the Introduction, this is true in any aligned space.

CoROLlaRy. For an ordered space $X$ with the order alignment,

$$
\bar{p}_{m}(X)=\bar{h}_{m}(X)+1 \text {. }
$$

Proof of Theorem 12. In light of the above corollary, it suffices to show that any subset $S$ of $X$ with core $_{m-1}(S)=\varnothing$ can contain at most $(m-1)(h-1)+1$ points. Since the order alignment is obviously extremally detachable, it suffices, by Theorem 8 , to show this in case $S$ is order convex. If $S$ is convex, then its Helly number in the order alignment can be no more than that of $X$. If, in fact, $h(S) \leqq h-1$, then we are done by (3). Thus $h(S)=h$ without loss of generality, so we may in fact assume $S=X$.

For each $s$ in $S$, let

$$
\nu(s)=|\downarrow s|-|\uparrow s|,
$$

and set

$$
\begin{aligned}
& P=\{s \in S: \nu(s) \geqq 0\}, \\
& N=\{s \in S: \nu(s) \leqq 0\}, \\
& Z=\{s \in S: \nu(s)=0\} .
\end{aligned}
$$

Note that $\nu$ is strictly monotone: $s<t$ implies $\nu(s)<\nu(t)$.

Let $A$ be the set of minimal elements of $P$ that are not in $Z$, and let $B$ be the set of maximal elements of $N$ that are not in $Z$. Then $A \cup Z$ and $B \cup Z$ are antichains and their union $A \cup B \cup Z$ is therefore independent in the order alignment. This union is also convex. For if $a>x>b$, then $\nu(a)>\nu(x)>\nu(b)$, so that $a$ cannot be minimal in $P$ if $\nu(x) \geqq 0$ whereas $b$ cannot be maximal in $N$ if $\nu(x) \leqq 0$. Thus $A \cup B \cup Z$ is free, so that

$$
h \geqq|A \cup B \cup Z|=|A|+|B|+|Z| .
$$

Now let $A^{\prime}$ be the set of minimal elements of $P \backslash(A \cup Z)$ and let $B^{\prime}$ be the maximals of $N \backslash(B \cup Z)$. Then $A^{\prime}$ and $B^{\prime}$ are antichains, and the two unions $A^{\prime} \cup A \cup Z$ and $B^{\prime} \cup B \cup Z$ are both free. Whence

$$
h \geqq\left|A^{\prime}\right|+|A|+|Z|
$$

and

$$
h \geqq\left|B^{\prime}\right|+|B|+|Z| \text {. }
$$


Note that for any $p$ in $P,|\uparrow p| \leqq m-1$ since otherwise $|\downarrow p| \geqq$ $|\uparrow p| \geqq m$, which would put $p$ in $\operatorname{core}_{m-1}(S)$ by Proposition 13. Now any $q$ in $A^{\prime}$ is strictly larger than some $p$ in $A \cup Z$. (Here we need the points of $S$ to be distinct.) Hence $|\uparrow q|<|\uparrow p| \leqq m-1$. That is $|\uparrow q| \leqq m-2$ for each $q$ in $A^{\prime}$. Analogous remarks hold for $N$ and $B^{\prime}$.

It is now time to distinguish four cases, depending on whether each of $A \cup Z$ and $B \cup Z$ contain more or less than $h / 2$ elements. Because $h$ is odd, a set either contains at most $(h-1) / 2$ elements or at least $(h+1) / 2$ elements.

Case I.

$$
\begin{aligned}
& |A|+|Z| \leqq(h-1) / 2 \\
& |B|+|Z| \leqq(h-1) / 2 .
\end{aligned}
$$

By choice of the various sets involved,

$$
S=\uparrow A \cup \uparrow Z \cup \downarrow B \cup \downarrow Z
$$

where $\uparrow A$ denotes the set of points which equal or lie above points in $A$, etc. Thus

$$
\begin{aligned}
|S| & \leqq|\uparrow A|+|\uparrow Z|+|\downarrow B|+|\downarrow Z| \\
& \leqq|A|(m-1)+|Z|(m-1)+|B|(m-1)+|Z|(m-1) \\
& \leqq(h-1)(m-1)
\end{aligned}
$$

as desired.

Case II.

$$
\begin{aligned}
& |A|+|Z| \geqq(h+1) / 2 \\
& |B|+|Z| \geqq(h+1) / 2 .
\end{aligned}
$$

Then $\left|A^{\prime}\right| \leqq(h-1) / 2$ and $\left|B^{\prime}\right| \leqq(h-1) / 2$, so that

$$
\begin{aligned}
|S| & \leqq\left|\uparrow A^{\prime}\right|+\left|\downarrow B^{\prime}\right|+|A|+|B|+|Z| \\
& \leqq\left|A^{\prime}\right|(m-2)+\left|B^{\prime}\right|(m-2)+h \\
& \leqq(h-1)(m-2)+h-1+1 \\
& \leqq(h-1)(m-1)+1
\end{aligned}
$$

as desired.

Case III.

$$
\begin{aligned}
& |A|+|Z| \geqq(h+1) / 2 \\
& |B|+|Z| \leqq(h-1) / 2 .
\end{aligned}
$$

Then $\left|A^{\prime}\right| \leqq(h-1) / 2$, so that 


$$
\begin{aligned}
|S| & \leqq\left|\uparrow A^{\prime}\right|+|A|+|\downarrow B|+|\downarrow Z| \\
& \leqq\left|A^{\prime}\right|(m-2)+|A|+|B|(m-1)+|Z|(m-1) \\
& \leqq\left(\left|A^{\prime}\right|+|B|+|Z|\right)(m-2)+|A|+|B|+|Z| \\
& \leqq(h-1)(m-2)+h \\
& \leqq(h-1)(m-1)+1
\end{aligned}
$$

as desired.

The fourth case is dual to Case III, thus completing the proof.

It is worth observing that the Helly number and rank of a partially ordered set $X$ are closely connected by means of the width $w(X)$ of $X$-the supremum of the cardinalities, of antichains in $X$. Indeed, any antichain together with a point not in it form a set with empty 1-core. Thus

$$
w(X)+1 \leqq h(X),
$$

(unless $X$ itself is an antichain in which case $w(X)=h(X)$ ). On the other hand, every independent set is easily seen to be the union of two antichains, whence

$$
\operatorname{rank}(X) \leqq 2 w(X) .
$$

Putting these inequalities together, we obtain

$$
w(X)+1 \leqq h(X) \leqq \operatorname{rank}(X) \leqq 2 w(X) .
$$

It is not difficult to construct examples illustrating the full range of possibilities in the above inequality.

When the rank and Helly number happen to be equal, then of course (8) is a consequence of the simple Proposition 3. We shall conclude by showing that this is the case for Pow $(n)$-the set of subsets of an $n$ element set ordered by inclusion.

Proposition 14. In Pow (n), the rank and the Helly number are both equal to mbe $(n+1)$.

Proof. In Pow $(n)$ the middle two layers (the sets of size $[n / 2]$ and $[n / 2]+1)$ form a clique in the order alignment of cardinality $\operatorname{mbc}(n+1)$. Thus $h$ (Pow $(n)) \geqq \operatorname{mbc}(n+1)$. To conclude the proof we need only show that rank (Pow $(n)) \leqq \operatorname{mbc}(n+1)$.

Let $\mathscr{I}$ be any order independent family. Let $\mathscr{A}$ be the antichain of maximal sets in $\mathscr{F}^{\prime}$ and $\mathscr{B}$ the antichains of minimal sets in $\mathscr{F}$. Since $p$ is independent, $\mathscr{J}=\mathscr{A} \cup \mathscr{B}$. Now let $p$ be a new point not in $X$, and define 


$$
\mathscr{B}^{\prime}=\{B \cup p: B \in \mathscr{B}\}
$$

Then $\mathscr{A} \cup \mathscr{B}^{\prime}$ is an antichain in Pow $(n+1)$ and hence has at most mbc $(n+1)$ members by Sperner's theorem.

\section{REFERENCES}

1. P. W. Bean, Helly and Radon-type theorems in interval convexity spaces, Pacific J. Math., 51 (1974), 363-368.

2. P. M. Cohn, Universal Algebra, Harper and Row, New York, 1965.

3. J.P. Doignon, Convexity in cristallographic lattices, J. Geometry, 3 (1973), 71-85.

4. J. Eckhoff, Der Satz von Radon in konvexen Produktstrukturen II, Monatsh. Math., 73 (1969), 7-30.

5. - Radon's theorem revisited, in Contributions to Geometry, Proc. Geom. Symp. Siegen 1978, J. Tölke and J. Wills, ed., Birkhäuser, Basel, 1979.

6. F. Harary, Graph Theory, Addison-Wesley, London 1969, 29.

7. R. E. Jamison, $A$ development of axiomatic convexity, Math. Tech. Report \#48, Clemson, S.C., 1970.

8. - A general theory of convexity, Dissertation, Univ. of Washington, Seattle, 1974.

9. Connectedness as convexity, in preparation.

10. D. C. Kay and E. W. Womble, Axiomatic convexity theory and relationship between the Carthéodory, Helly, and Radon numbers, Pacific J. Math., 38 (1971), 471-485.

11. F. W. Levi, On Helly's theorem and the axioms of convexity, J. Indian Math. Soc., (N.S.) 15 (1951), 65-76.

12. J. Radon, Mengen konvexer Körper, die einen gemeinsamen Punkt enthalten, Math. Ann., 83 (1921), 113-115.

13. G. Sierksma, Carathéodory and Helly numbers of convex product structures, Pacific J. Math., 61 (1975), 275-282.

14. G. Sierksma and J.R. Reay, A Tverberg-type generalization of the Helly number of a convexity space, Math. Preprint, Western Wash. University, Bellingham, 1979.

15. G. Thompson and W. R. Hare, Tverberg-type theorems in convex product structures, Springer Lect. Notes Math., 490 (1975), 212-217.

16. H. Tverberg, A generalization of Radon's theorem, J. London Math. Soc., 41 (1966), 123-128.

\section{Appendix \\ Bivalent semilattices}

A (meet) semilattice is a partially ordered set in which any pair of elements has an infimum. It is customary to write $x y$ for the infimum of $x$ and $y$. (This is a reminder of the fact that a semilattice may also be defined as a commutative idempotent semigroup. The two definitions are related by the observation that $x \leqq y$ is equivalent to $x=x y$.) The family $\mathscr{S}$ of all subsemilattices of a semilattice $S$ forms the semilattice alignment on $S$.

Although the determination (given below) of the Helly number of a semilattice alignment is quite easy, the evaluation of the partition numbers seems, in general, to be rather difficult. One nice 
result of this kind which confirms the Partition Conjecture for the join semilattice $\mathrm{Pow}_{1}(n)$ of nonempty subsets of an $n$ element set is contained in the results of Lindstrom [A1] and Tverberg [A2]:

$$
p_{m}\left(\operatorname{Pow}_{1}(n)\right)=(m-1) n+1 .
$$

The Main Theorem can be applied to establish the Partition Conjecture for another (unfortunately rather limited) class of semilattices.

By the valence of a point $p$ in an aligned space $X$, we shall mean the number of copoints at $p$. (In trees this coincides with the graph notion of valence.) The valence val $(X)$ of $X$ is taken to be the supremum of the valences of the points of $X$. Notice that $\operatorname{val}(X) \leqq n$ can be expressed by saying that $X$ satisfies $\operatorname{CIP}(n+1,1)$. (Thus any space of valence $n$ has Carathéodory number at most $n$ by Proposition 6.) In particular, if $X$ is bivalent (val $(X) \leqq 2$ ), then $X$ has CIP $(3,2)$ and the Main Theorem applies. In the last section, we observed that an ordered set with the order alignment is always bivalent.

A point $x$ of an ordered set $X$ is said to cover $z$ in $X$ provided $x>z$ and there is no $y$ in $X$ with $x>y>z$. If $p$ is a nonmaximal point in finite meet semilattice $S$, one can easily check that each copoint at $p$ (w.r.t. the semilattice alignment on $S$ ) has the form

$$
\uparrow c \cup(S \backslash \uparrow p),
$$

where $c$ covers $p$. Furthermore, for each cover $c$ of $p$, the above set is a copoint at $p$. Thus the valence of $p$ is just the number of covers of $p$ in $S$. (Any maximal element of $S$ is an extreme point and hence has a unique copoint-namely, its complement.) Thus a finite semilattice $S$ is bivalent iff each point of $S$ is covered by at most two points of $S$. The diagrams below illustrate bivalent semilattices.
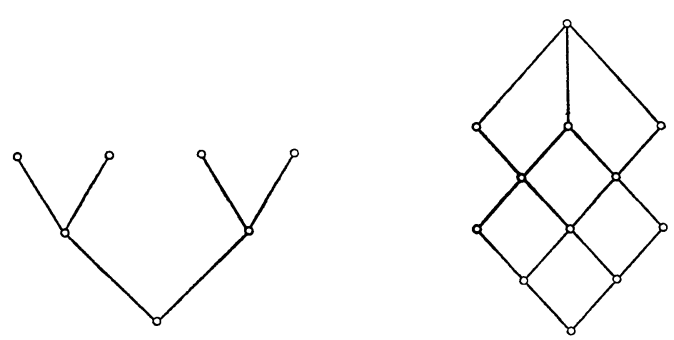

To evaluate the partition numbers of bivalent semilattices, it remains only to determine their Helly numbers. This could be done by applying Theorem 7: it is easily seen that the semilattice alignment 
is $\mathrm{ED}$ and that the cliques are just the maximal chains. However, it is possible to sharpen the estimates of the restricted Helly numbers and hence obtain better estimates for $\bar{p}_{m}$. Recall that the length $l(X)$ of an ordered set $X$ is customarily defined to be one less than the supremum of the cardinalities of the chains in $X$.

Proposition 15. Let $S$ be a semilattice of finite length $l(S)$. The restricted Helly numbers for the semilattice alignment $\mathscr{S}$ on $S$ satisfy

$$
\bar{h}_{m}(S) \leqq m l(S)+1
$$

Proof. Let $A$ be a subset of $S$ with $|A|>m l(S)+1$. We shall show by induction on $l(S)$ that core $_{m}(A) \neq \varnothing$.

For $l(S)=1$, note that any two points of $A$ generate the unique minimum of $S$. (Here the distinctness of the points of $A$ is used.) Since $|A| \geqq m+2$, it follows that $\inf (S)$ lies in core $_{m}(A)$.

Proceeding inductively, consider any $B \subset A$ with $|B| \leqq m$. If $\inf (A \backslash B) \neq \inf (A)$, then $\inf (A \backslash B)>\inf (A)$ so that

$$
l(\mathscr{S}(A \backslash B))<l(\mathscr{S}(A)) \leqq l(S) .
$$

Since $|A \backslash B| \geqq m(l(S)-1)+2$, it follows by induction that core ${ }_{m}(A \backslash B)$ -and hence the larger set $\operatorname{core}_{m}(A)$-is not empty. If however, $\inf (A \backslash B)=\inf (A)$ for all $B \subset A$ with $|B| \leqq m$, then inf $(A)$ lies in core $_{m}(A)$.

That this result is the best possible in general is seen in the example pictured below. The $l m+1$ maximal elements have empty $m$-core.

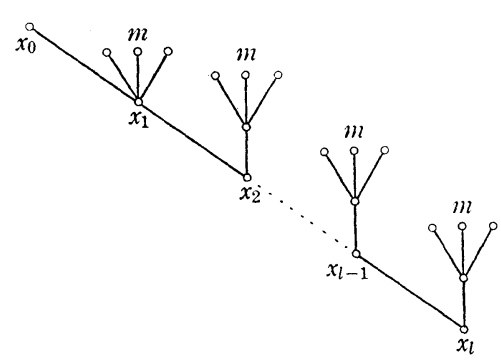

Applying the strong version of the Main Theorem quoted in the beginning of its proof, we obtain the following result.

THEOREM 15. Let $S$ be a bivalent semilattice of finite length 2 or more. Then the partition numbers of the semilattice alignment on $S$ satisfy 


$$
\begin{aligned}
& p_{m}(S)=(m-1)(l(S)+1)+1, \\
& \bar{p}_{m}(S) \leqq(m-1) l(S)+2 .
\end{aligned}
$$

Thus we have another instance in which the restricted partition numbers are strictly less than their unrestricted analogues.

A more thorough discussion of partition numbers in semilattices will appear separately. It seems worthwhile, however, to mention here one weak but generally valid bound.

Proposition 16. If $S$ is any semilattice of finite length, then

$$
p_{m}(S) \leqq(m-1)(l(S)+1)^{2}-l(S)+1 \text {. }
$$

Proof. One checks that the Carathéodory number of the semilattice alignment (commonly called the "breadth" of the semilattice) is bounded by the length plus one. The result then follows from Theorem 2.

Note added in proof. Doignon's theorem was also discovered independently by H. E. Scarf (Proc. Natl. Acad. Sci. USA 74 (1977), 3637-3641) in the context of integer programming. Seeking an abstract basis for Scarf's result, A. J. Hoffman (Annals of NY Acad. Sci. 319 (1979), 284-288) was led to an independent discovery of Theorem 7 above.

\section{REFERENCES FOR THE APPENDIX}

A1. B. Lindstrom, A theorem on families of sets, J, Combinatorial Theory-(A) 13 (1972), 274-277.

A2. H. Tverberg, On equal unions of sets, Studies in Pure Math. (ed. L. Mirski), Academic Press, London, (1971), 249-250.

Received November 19, 1979. The author wishes to express his thanks to the Arbeitsgruppe 1 im Fachbereich Mathematik of the Technische Hochschule Darmstadt for their support during the preparation of this paper.

Technische Hochschule Darmstadt

D-6100 DARMSTADT

AND

Clemson University

Clemson, SC 29631 


\section{PACIFIC JOURNAL OF MATHEMATICS}

\section{EDITORS}

DONALD BABBITT (Managing Editor)

University of California

Los Angeles, California 90024

Hugo RossI

University of Utah

Salt Lake City, UT 84112

C. C. MOORE and ANDREW OGG

University of California

Berkeley, CA 94720

\section{J. DugundJI}

Department of Mathematics University of Southern California Los Angeles, California 90007

R. FinN and J. Milgram Stanford University Stanford, California 94305

\section{ASSOCIATE EDITORS}
R. ARENS
E. F. BECKENBACH
B. H. Neumann
F. WOLF
K. YoshidA

\section{SUPPORTING INSTITUTIONS}

UNIVERSITY OF ARIZONA

UNIVERSITY OF BRITISH COLUMBIA

CALIFORNIA INSTITUTE OF TECHNOLOGY

UNIVERSITY OF CALIFORNIA

MONTANA STATE UNIVERSITY

UNIVERSITY OF NEVADA, RENO

NEW MEXICO STATE UNIVERSITY

OREGON STATE UNIVERSITY
UNIVERSITY OF OREGON

UNIVERSITY OF SOUTHERN CALIFORNIA

STANFORD UNIVERSITY

UNIVERSITY OF HAWAII

UNIVERSITY OF TOKYO

UNIVERSITY OF UTAH

WASHINGTON STATE UNIVERSITY

UNIVERSITY OF WASHINGTON 


\section{Pacific Journal of Mathematics}

\section{Vol. 96, No. $1 \quad$ November, 1981}

Hédi Amara, Groupe des classes et unité fondamentale des extensions quadratiques relatives à un corps quadratique imaginaire principal $\ldots \ldots \ldots 1$

Douglas S. Bridges, On the isolation of zeroes of an analytic function $\ldots \ldots 13$ Andrew J. Casson and John L. Harer, Some homology lens spaces which

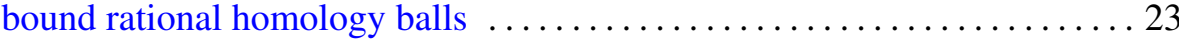

Z. A. Chanturia, On the absolute convergence of Fourier series of the classes $H^{\omega} \cap V[v]$

J.-F. Colombeau and Mário Carvalho Matos, On some spaces of entire functions defined on infinite-dimensional spaces $\ldots \ldots \ldots \ldots \ldots \ldots \ldots 63$

Edwin Duda, Pointwise periodic homeomorphisms on chainable continua . . .77

Richard F. Gustafson, A simple genus one knot with incompressible spanning surfaces of arbitrarily high genus $\ldots \ldots \ldots \ldots \ldots \ldots \ldots \ldots 1$

Fumio Hiai, Masanori Ohya and Makoto Tsukada, Sufficiency, KMS condition and relative entropy in von Neumann algebras

Ted Hurley, Intersections of terms of polycentral series of free groups and free Lie algebras. II .................................. 111

Robert Edward Jamison, II, Partition numbers for trees and ordered sets . . 115 R. D. Ketkar and N. Vanaja, A note on FR-perfect modules ............. 141 Michihiko Kikkawa, On Killing-Ricci forms of Lie triple algebras ....... 153

Jorge Lewowicz, Invariant manifolds for regular points 163

Richard W. Marsh, William H. Mills, Robert L. Ward, Howard Rumsey and Lloyd Richard Welch, Round trinomials .....

Claude Schochet, Topological methods for $C^{*}$-algebras. I. Spectral sequences

Yong Sian So, Polynomial near-fields?

Douglas Wayne Townsend, Imaginary values of meromorphic functions in the disk

Kiyoshi Watanabe, Coverings of a projective algebraic manifold .. 243

Martin Michael Zuckerman, Choosing $l$-element subsets of $n$-element sets 